\title{
On linear extendability of isometrical embeddings
}

\author{
Hossein Khodaiemehr $\quad$ Fereshteh Sady
}

\begin{abstract}
In this paper we first investigate linear extendability of an isometric embedding $T: \mathcal{U} \longrightarrow \mathcal{Y}$ from an open subset $\mathcal{U}$ of a real Banach space $\mathcal{X}$ into a real Banach space $\mathcal{Y}$ in the case where $\mathcal{Y}$ is either the space $C_{\mathbb{R}}(K)$ of continuous real-valued functions on a compact space $K$, or is a strictly convex Banach space. Then we obtain similar results for the case where $\mathcal{Y}$ is an arbitrary real Banach space and $T: \mathcal{U} \longrightarrow \mathcal{Y}$ is an isometry whose range satisfies some additional conditions.
\end{abstract}

\section{Introduction}

The problem of determining conditions under which an isometry between two normed linear spaces is affine has been studied by many authors and has its roots in the famous Mazur-Ulam Theorem [10]. By the Mazur-Ulam theorem every surjective isometry between two real normed spaces is affine. Using the idea of [18], a surprisingly short proof of Mazur-Ulam theorem has been given by J. Väisälä in [16]. This theorem has been generalized by many authors by relaxing the surjectivity assumption. For instances, in [3], Figiel proved that for an isometry $T: \mathcal{X} \longrightarrow \mathcal{Y}$ between real normed spaces $\mathcal{X}$ and $\mathcal{Y}$, there exists a linear map $S: \mathcal{Y} \longrightarrow \mathcal{X}$ such that $S(T(x))=x$ for all $x \in \mathcal{X}$ and moreover the restriction of $S$ to the linear span of $T(X)$ has norm 1. In [4], the surjectivity assumption was replaced by a weaker assumption that every direction in $\mathcal{Y}$ can

Received by the editors in November 2015.

Communicated by F. Bastin.

2010 Mathematics Subject Classification : Primary 46J10, 47B48; Secondary 46J20.

Key words and phrases : Mazur-Ulam theorem, Isometry, Strictly convex, Isometries, reallinear isometry. 
be approximated by a direction between two points in the range of the isometry $T$. In [1] Baker proved that every isometry from a normed space into a strictly convex normed linear space is affine. On the other hand, in [17] it was shown that for a real Banach space $X$ and a compact Hausdorff space $K$, if $T: X \rightarrow C_{\mathbb{R}}(K)$ is an isometry with $T(0)=0$, then there exists a closed nonempty subset $L \subseteq K$, such that the superposition $Q \circ T: X \rightarrow C_{\mathbb{R}}(L)$ is a linear map. Here $C_{\mathbb{R}}(K)$ is the space of all real-valued continuous functions on $K$ and $Q: C_{\mathbb{R}}(K) \longrightarrow C_{\mathbb{R}}(L)$ is the restriction map. For some related results, we may refer to [18] and [12]. By introducing the notion of metricoid spaces, a generalization of Mazur-Ulam theorem for isometries between metricoid spaces was given in [7].

In [9] Mankiewiz considered the extension problem of isometries whose domains are subsets of normed spaces and proved that every surjective isometry between open connected subsets of normed spaces can be extended to a surjective affine isometry between normed spaces. The extension problem of isometries on unit spheres of some normed spaces has been considered for examples in $[8,15,19,20]$

Recently, by proving some Mazur-Ulam type theorems, in [5] and [6], Hatori investigated the problem of linear extendability of surjective isometries between open subgroups (or certain open subsets) of invertible elements of unital Banach algebras see [5, Theorem 3.2] and [6, Theorem 3.1].

Motivated by the Backer's and Villa's results concerning (into) isometries, and Harori's work on linear extendability of surjective isometries between open subgroups of invertible elements in Banach algebras, in this paper we first investigate linear extendability of an isometry from a certain open subset $\mathcal{U}$ of a Banach space $\mathcal{X}$ into a Banach space $\mathcal{Y}$, in the case where $\mathcal{Y}$ is either the space $C_{\mathbb{R}}(K)$, for a compact Hausdorff space $K$, or a strictly convex Banach space. In the first case we show that there exists a closed subset $L \subseteq K$ and a real-linear isometry $\widetilde{T}: \mathcal{X} \longrightarrow C_{\mathbb{R}}(L)$ which extends $Q \circ T$ (up to a translation), where $Q: C_{\mathbb{R}}(K) \longrightarrow C_{\mathbb{R}}(L)$ is the restriction map, and in the second case where $\mathcal{Y}$ is strictly convex, it turns out that such an isometry $T: \mathcal{U} \longrightarrow \mathcal{Y}$ extends to a real-linear isometry from $\mathcal{X}$ into $\mathcal{Y}$ up to a translation.

Imposing some additional assumptions on the range of a given isometry $T: \mathcal{U} \longrightarrow \mathcal{Y}$, where $\mathcal{Y}$ is an arbitrary Banach space, we obtain similar results for this case (Theorems 3.6 and 3.8).

\section{Preliminaries}

Let $\mathcal{X}$ and $\mathcal{Y}$ be normed spaces and $\mathcal{U}$ be a subset of $\mathcal{X}$. A mapping $T: \mathcal{U} \longrightarrow \mathcal{Y}$ is called an isometrical embedding or an isometry if $\|T u-T v\|=\|u-v\|$ for all $u, v \in \mathcal{U}$.

As we mentioned before, Mazur-Ulam Theorem states that any surjective isometry $T: \mathcal{X} \longrightarrow \mathcal{Y}$ between real normed spaces $\mathcal{X}$ and $\mathcal{Y}$ is real-linear up to a translation, that is, $T-T 0$ is real-linear. A normed linear space $\mathcal{Y}$ is said to be strictly convex, provided that for each $a, b \in \mathcal{Y}$ the equality $\|a+b\|=\|a\|+\|b\|$ implies $\{a, b\}$ is linearly dependent, or equivalently for nonzero elements $a, b \in \mathcal{Y}$, the equality $\|a+b\|=\|a\|+\|b\|$ implies that $a=t b$ for some $t>0$. For instances, 
inner product spaces as well as $\ell_{p}$ spaces for $1<p<\infty$ are strictly convex. By Baker's result [1], every isometrical embedding from a normed space into a strictly convex normed space is affine. We recall that in a strictly convex normed space $\mathcal{Y}$, by [1], for each $a, b \in \mathcal{Y}, \frac{1}{2}(a+b)$ is the unique point in $\mathcal{Y}$ with distance equal to $\frac{1}{2}\|a-b\|$ from both $a$ and $b$.

In a normed space $\mathcal{X}$, a point $a$ of the sphere $S_{r}=\{x \in \mathcal{X}:\|x\|=r\}, r>0$, is called smooth if there exists a unique $f_{a} \in \mathcal{X}^{*}$ such that $f_{a}(a)=\|a\|$ and $\left\|f_{a}\right\|=1$. Mazur proved that the set of all smooth points in a separable Banach space $\mathcal{X}$ is a dense $G_{\delta}$ subset of $\mathcal{X}$ (see [13, Proposition 9.4.3]).

For a compact Hausdorff space $K$ we denote the Banach space of real-valued, respectively complex-valued continuous functions on $K$ by $C_{\mathbb{R}}(K)$ and $C(K)$ endowed with the supremum norm $\|\cdot\|_{K}$. For each $t \in K$, the evaluation functional at $t$ defined on these spaces will be denoted by $\delta_{t}$. For each continuous function $f$ on $K$ we denote the maximum set of modulus of $f$ by $M(f)$, that is $M(f)=\left\{x \in K:|f(x)|=\|f\|_{K}\right\}$.

Let $\mathcal{X}$ be a real or complex normed space and $\mathcal{X}_{1}^{*}$ be the closed unit ball of $\mathcal{X}^{*}$ endowed with the weak-star topology. Let $i_{\mathcal{X}}$ be the isometrical embedding from $\mathcal{X}$ to $C_{\mathbb{R}}\left(\mathcal{X}_{1}^{*}\right)$ or $C\left(\mathcal{X}_{1}^{*}\right)$ defined by $i_{\mathcal{X}}(x)(l)=l(x)$, for $l \in \mathcal{X}_{1}^{*}$ and $x \in \mathcal{X}$. There are some real or complex normed spaces $\mathcal{X}$ containing an element $e_{0}$ such that for each $x \in \mathcal{X}$, the equality $i_{\mathcal{X}}(x)=0$ on the maximum set $M\left(i_{\mathcal{X}}\left(e_{0}\right)\right)$ of $i_{\mathcal{X}}\left(e_{0}\right)$ implies that $x=0$. Clearly if $A$ is a commutative semisimple unital complex Banach algebra with unit element $e$, then the maximum set $M\left(i_{A}(e)\right)$ has this property. More generally, if $B$ is a unital complex Banach algebra, $A$ is a closed semisimple commutative subalgebra of $B$ containing the unit of $B$ and $x_{0}$ is an invertible element of $B$ with $\left\|x_{0}\right\|=\left\|x_{0}^{-1}\right\|=1$ (for example unitary elements in $C^{*}$-algebras satisfy these conditions), then for the complex normed space $\mathcal{X}=A x_{0}$, the element $x_{0} \in \mathcal{X}$ has the mentioned property. Indeed, for each complex homomorphism $\varphi$ on $A$, it is easy to see that the map $\widetilde{\varphi} \in \mathcal{X}^{*}$ defined by $\tilde{\varphi}\left(x x_{0}\right)=\varphi(x), x \in A$, is an element of the unit ball of $\mathcal{X}^{*}$ with $\widetilde{\varphi}\left(x_{0}\right)=1$. Hence, since the maximum set $M\left(i_{\mathcal{X}}\left(x_{0}\right)\right)$ of $i_{\mathcal{X}}\left(x_{0}\right)$ contains all $\widetilde{\varphi}$, it follows that for each $x \in A$ with $i_{\mathcal{X}}\left(x x_{0}\right)=0$ on $M\left(i_{\mathcal{X}}\left(x_{0}\right)\right)$ we have $\varphi(x)=0$ for all complex homomorphisms $\varphi$ on $A$ and hence $x=0$.

\section{Main Results}

Given real normed spaces $\mathcal{X}, \mathcal{Y}$ and an isometrical embedding $T: \mathcal{U} \longrightarrow \mathcal{Y}$ where $\mathcal{U}$ is a certain subset of $\mathcal{X}$, we investigate the extendability of $T$ to a linear isometry between $\mathcal{X}$ and $\mathcal{Y}$. We first consider the case where $\mathcal{Y}=C_{\mathbb{R}}(K)$, for some compact Hausdorff space $K$ and then we investigate similar problem for the case that the range of $T$ is contained in a strictly convex real Banach space $\mathcal{Y}$ or satisfies a property as a subset of an arbitrary real Banach space $\mathcal{Y}$.

The motivation of considering isometrical embeddings to $C_{\mathbb{R}}(K)$ comes from Villa's result [17] and Hatori's result [5] and in the proofs we make use the ideas of these papers. For stating the result, we need the following lemma concerning smooth points of the spheres in Banach spaces. 
Lemma 3.1. Let $\mathcal{X}, \mathcal{Y}$ be real Banach spaces and $\mathcal{V}$ be a nonempty closed subset of $\mathcal{X}$ such that $\mathbb{R} \mathcal{V} \subseteq \mathcal{V}$. Let $T: \mathcal{V} \rightarrow \mathcal{Y}$ be an isometric embedding satisfying $T(0)=0$. If $a \in \mathcal{V}$ is a smooth point of the sphere $S_{\|a\|}$ and $f \in \mathcal{Y}^{*}$ is a linear functional of norm one, such that for all real $r \in \mathbb{R}$,

$$
f(T(r a))=r\|a\|
$$

then $f(T(x))=f_{a}(x)$ holds for all $x \in \mathcal{V}$.

Proof. The proof is a minor modification of [13, Lemma 9.4.6]

Theorem 3.2. Let $\mathcal{X}$ be a real Banach space and $\mathcal{U}$ be a nonempty open subset of $\mathcal{X}$ such that $\mathbb{R} \backslash\{0\} \mathcal{U} \subseteq \mathcal{U}$. Then for each isometry $T: \mathcal{U} \rightarrow\left(C_{\mathbb{R}}(K),\|.\|_{K}\right)$, where $K$ is a compact Hausdorff space, there exists a nonempty and closed subset $L \subseteq K$, a function $g \in C_{\mathbb{R}}(K)$ and a linear isometry $\widetilde{T}: \mathcal{X} \rightarrow\left(C_{\mathbb{R}}(L),\|.\|_{L}\right)$ such that $\widetilde{T}(u)=\left.(T(u)-g)\right|_{L}$ holds for all $u \in \mathcal{U}$.

Proof. Fixing a point $e_{0} \in \mathcal{U}$, there exists $r_{0}>0$ such that the closed ball $B_{r_{0}}\left(e_{0}\right)=$ $\left\{x \in \mathcal{U}:\left\|x-e_{0}\right\| \leq r_{0}\right\}$ is contained in $\mathcal{U}$. Since, by hypothesis $\mathbb{R} \backslash\{0\} \mathcal{U} \subseteq \mathcal{U}$ replacing $e_{0}$ by $\frac{1}{r_{0}} e_{0}$ we may assume that $r_{0}=1$ and consequently the convex subset $\mathbb{R}^{+} B_{1}\left(e_{0}\right)=\left\{r x: r>0, x \in B_{1}\left(e_{0}\right)\right\}$ is contained in $\mathcal{U}$. We note that the sequence $\left\{\frac{e_{0}}{n}\right\}$ of elements of $\mathcal{U}$ converges to 0 and since $T$ is an isometry on $\mathcal{U}$, $\left\{T\left(\frac{e_{0}}{n}\right)\right\}$ is a cauchy sequence in $C_{\mathbb{R}}(K)$ and so converges to some $g \in C_{\mathbb{R}}(K)$. It is now easy to see that the map $S: \mathcal{U} \cup\{0\} \longrightarrow \mathcal{Y}$ defined by $S(u)=T(u)-g$ for $u \in \mathcal{U}$ and $S(0)=0$ is an isometry, as well. Hence replacing $\mathcal{U}$ by $\mathcal{U} \cup\{0\}$ and $T$ by $S$ we may assume that $\mathcal{U}$ contains zero and $T(0)=0$ (with this assumption that $\mathcal{U} \backslash\{0\}$ is open in $\mathcal{X}$ ).

Let $\mathcal{V}$ denote the norm closure of $\mathcal{U}$ in $\mathcal{X}$. Being an isometry, we can extend $T$ to an isometry, denoted again by $T$ from $\mathcal{V}$ into $C_{\mathbb{R}}(K)$. We now define the map $T_{1}: \mathcal{X} \rightarrow C_{\mathbb{R}}(K)$ by

$$
T_{1}(a)= \begin{cases}0 & a=0 \\ T\left(a+\|a\| e_{0}\right)-T\left(\|a\| e_{0}\right) & a \neq 0\end{cases}
$$

Clearly $T_{1}$ is well-defined, since for each $a \in \mathcal{X},\|a\| e_{0}, a+\|a\| e_{0} \in\|a\| B_{1}\left(e_{0}\right) \subseteq \mathcal{U}$.

We first claim that for each point $a \in \mathcal{X}$, we have $\bigcap_{r \in \mathbb{R}} M(T(r a)) \neq \varnothing$, where, as we mentioned before, $M(f)$ is the maximum set $\left\{t \in K:|f(t)|=\|f\|_{K}\right\}$ of a continuous function $f$ on $K$. To prove the claim, it suffices to show that the family $\{M(T(r a): r \in \mathbb{R}\}$ of compact subsets of $K$ has finite intersection property. Let $r_{1}, \ldots, r_{n} \in \mathbb{R}$ be arbitrary. Without loss of generality we may assume that $r_{1} \leq$ $r_{2} \leq \ldots \leq r_{n}$ and $r_{i}=0$ for some $1 \leq i \leq n$. Then for each $t \in M\left(T\left(r_{n} a\right)-T\left(r_{1} a\right)\right)$ if $i \leq j \leq n$ then

$$
\begin{aligned}
\left(r_{n}-r_{1}\right)\|a\| & =\left\|T\left(r_{n} a\right)-T\left(r_{1} a\right)\right\|_{K}=\left|\left(T\left(r_{n} a\right)-T\left(r_{1} a\right)\right)(t)\right| \\
& \left.\leq\left|\left(T\left(r_{n} a\right)-T\left(r_{j} a\right)\right)(t)\right|+\left|T\left(r_{j} a\right)(t)\right|+\mid T\left(r_{1} a\right)\right)(t) \mid \\
& \leq\left\|T\left(r_{n} a\right)-T\left(r_{j} a\right)\right\|_{K}+\left\|T\left(r_{j} a\right)\right\|_{K}+\left\|T\left(r_{1} a\right)\right\|_{K} \\
& =\left(r_{n}-r_{j}\right)\|a\|+r_{j}\|a\|-r_{1}\|a\|=\left(r_{n}-r_{1}\right)\|a\|
\end{aligned}
$$


which shows that $\left|T\left(r_{j} a\right)(t)\right|=r_{j}\|a\|=\left\|T\left(r_{j} a\right)\right\|_{K}$, that is $t \in M\left(T\left(r_{j} a\right)\right)$. Similarly if $1 \leq j \leq i$, then

$$
\begin{aligned}
\left(r_{n}-r_{1}\right)\|a\| & =\left\|T\left(r_{n} a\right)-T\left(r_{1} a\right)\right\|_{K}=\left|\left(T\left(r_{n} a\right)-T\left(r_{1} a\right)\right)(t)\right| \\
& \left.\leq\left|\left(T\left(r_{n} a\right)(t)|+| T\left(r_{j} a\right)\right)(t)\right|+\mid T\left(r_{j} a\right)(t)-T\left(r_{1} a\right)\right)(t) \mid \\
& \leq\left\|T\left(r_{n} a\right)\right\|_{K}+\left\|T\left(r_{j} a\right)\right\|_{K}+\left\|T\left(r_{j} a\right)-T\left(r_{1} a\right)\right\|_{K} \\
& =r_{n}\|a\|-r_{j}\|a\|+\left(r_{j}-r_{1}\right)\|a\|=\left(r_{n}-r_{1}\right)\|a\|
\end{aligned}
$$

which implies that $t \in M\left(T\left(r_{j} a\right)\right)$. Therefore, $t \in M\left(T\left(r_{j} a\right)\right)$ for all $1 \leq j \leq n$, as desired. Hence $\bigcap_{r \in \mathbb{R}} M(T(r a)) \neq \varnothing$, as we claimed.

Let $a \in \mathcal{V}$ be a smooth point of the sphere $S_{\|a\|}$ and $f_{a} \in \mathcal{X}^{*}$ be the functional of norm one with $f_{a}(a)=\|a\|$. By the above discussion, there exists $t_{a} \in K$ such that for each $r \in \mathbb{R}, T(r a)\left(t_{a}\right)=\epsilon_{a}^{r} r\|a\|$ for some $\epsilon_{a}^{r} \in\{-1,1\}$. Since for infinitely many $n \in \mathbb{Z}, \epsilon_{a}^{n}$ are all equal, it follows easily that there exists $\epsilon_{a} \in\{1,-1\}$ such that for each $r \in \mathbb{R}, T(r a)\left(t_{a}\right)=\epsilon_{a} r\|a\|$. Hence it follows from Lemma 3.1 that $f_{a}(x)=\varepsilon_{a} T(x)\left(t_{a}\right)$ for all $x \in \mathcal{V}$. Therefore, $f_{a}=\varepsilon_{a} \delta_{t_{a}} \circ T$ on $\mathcal{V}$, that is $\delta_{t_{a}} \circ T$ has a linear extension $\epsilon_{a} f_{a} \in \mathcal{X}^{*}$ (with norm 1 ).

We now consider the subset $L$ of $K$ as the closure of

$$
L_{0}=\left\{t \in K: \delta_{t} \circ T \text { has a linear extension on } \mathcal{X}\right\} .
$$

By the above argument, for every smooth point $a \in \mathcal{A}$ which belongs to $\mathcal{V}$, there exists $t_{a} \in L_{0}$ such that $\left|T(a)\left(t_{a}\right)\right|=\|a\|$. As in [17], we consider two cases:

Case 1: Assume first that $\mathcal{X}$ is separable.

We note that $\mathcal{V}=\overline{\mathcal{U} \backslash\{0\}}$, since 0 is in the closure of $\mathcal{U}$. Hence since $\mathcal{U} \backslash\{0\}$ is an open subset of $\mathcal{X}$ and the set of all smooth points of $\mathcal{X}$ is a dense $G_{\delta}$ subset of $\mathcal{X}$, it follows that the set of all smooth points of $\mathcal{X}$ contained in $\mathcal{U} \backslash\{0\}$ is a dense $G_{\delta}$ subset of $\mathcal{V}$, as well. Let $Q: C_{\mathbb{R}}(K) \longrightarrow C_{\mathbb{R}}(L)$ be the restriction map. Then the definition of $L$ shows that the equality

$$
Q \circ T(r v)=r Q \circ T(v)
$$

holds for all $r \in \mathbb{R}$ and $v \in \mathcal{V}$. Moreover, if $a, b \in \mathcal{V}$ such that $a+b \in \mathcal{V}$, then since for each $t \in L_{0}, \delta_{t} \circ T$ has a real-linear extension on $\mathcal{X}$ we have

$$
Q \circ T(a+b)=Q \circ T(a)+Q \circ T(b) .
$$

Hence for each $a \in \mathcal{U}$, since $a+\|a\| e_{0},-\|a\| e_{0} \in \mathcal{U} \subset \mathcal{V}$ we have

$$
Q \circ T_{1}(a)=Q \circ T\left(a+\|a\| e_{0}\right)-Q \circ T\left(\|a\| e_{0}\right)=Q \circ T(a)
$$

Setting $\widetilde{T}=Q \circ T_{1}$, the above equality shows that $\widetilde{T}(a)=Q \circ T(a)$ for all $a \in \mathcal{U}$.

Now we show that $\widetilde{T}$ is a linear isometry from $\mathcal{X}$ into $C_{\mathbb{R}}(L)$. To prove the linearity of $\widetilde{T}$, we apply a similar argument as in [5, Theorem 3.2]. Let $c$ be a nonzero element in $\mathcal{X}$. Then $c+r e_{0},\|c\| e_{0} \in \mathcal{U}$ and $c+(r+\|c\|) e_{0} \in \mathcal{U}$ for all $r \geq\|c\|$. Hence by (3.3)

$$
\begin{aligned}
Q \circ T\left(c+r e_{0}\right)+Q \circ T\left(\|c\| e_{0}\right)=Q \circ T\left(c+r e_{0}+\right. & \left.\|c\| e_{0}\right)= \\
& Q \circ T\left(c+\|c\| e_{0}\right)+Q \circ T\left(r e_{0}\right)
\end{aligned}
$$


and consequently by (3.4)

$$
\widetilde{T}(c)=Q \circ T\left(c+r e_{0}\right)-Q \circ T\left(r e_{0}\right) .
$$

We first show that $\widetilde{T}(a+b)=\widetilde{T}(a)+\widetilde{T}(b)$, for every $a, b \in \mathcal{X}$. Since clearly $\widetilde{T}$ is continuous we may assume without loss of generality that $a \neq 0, b \neq 0$ and $a+b \neq 0$. Since $\|a+b\| \leq\|a\|+\|b\|$, using (3.5) and (3.3) we have

$$
\begin{aligned}
\widetilde{T}(a+b) & =Q \circ T\left(a+b+\|a\| e_{0}+\|b\| e_{0}\right)-Q \circ T\left(\|a\| e_{0}+\|b\| e_{0}\right) \\
& =Q \circ T\left(a+\|a\| e_{0}\right)+Q \circ T\left(b+\|b\| e_{0}\right)-Q \circ T\left(\|a\| e_{0}\right)-Q \circ T\left(\|b\| e_{0}\right) \\
& =\widetilde{T}(a)+\widetilde{T}(b)
\end{aligned}
$$

Now we show that $\widetilde{T}(r a)=r \widetilde{T}(a)$ holds for all $a \in \mathcal{X}$ and $r \in \mathbb{R}$. If $a=0$ or $r=0$, then the equality is trivial. So assume that $a \neq 0$ and $r \neq 0$. The case where $r>0$ is immediate from the definition of $\widetilde{T}$ and equality (3.2). In the case where $r<0$, since $-a+\|a\| e_{0}, a+\|a\| e_{0}, 2\|a\| e \in \mathcal{U}$ it follows from (3.3) that

$$
\begin{aligned}
Q \circ T\left(-a+\|a\| e_{0}\right)+Q \circ T\left(a+\|a\| e_{0}\right) & =Q \circ T\left(2\|a\| e_{0}\right) \\
& =Q \circ T\left(\|a\| e_{0}\right)+Q \circ T\left(\|a\| e_{0}\right)
\end{aligned}
$$

and therefore

$$
\begin{aligned}
\widetilde{T}(r a) & =-r\left(Q \circ T\left(-a+\|a\| e_{0}\right)-Q \circ T\left(\|a\| e_{0}\right)\right) \\
& =-r\left(-Q \circ T\left(a+\|a\| e_{0}\right)+Q \circ T\left(\|a\| e_{0}\right)\right)=r \widetilde{T}(a) .
\end{aligned}
$$

which shows that $\widetilde{T}$ is linear.

Clearly for each point $a \in \mathcal{X}$,

$$
\begin{aligned}
\|\widetilde{T}(a)\|_{L} & =\|Q \circ T(a+\|a\| e)-Q \circ T(\|a\| e)\|_{L} \\
& \leq\|T(a+\|a\| e)-T(\|a\| e)\|_{K} \\
& =\|a+\| a\|e-\| a\|e\|=\|a\| .
\end{aligned}
$$

Now if $a \in \mathcal{X}$ is a smooth point and $t_{a} \in L_{0}$ and $f_{a} \in \mathcal{X}^{*}$ are as in the beginning of the proof, then since $a+\|a\| e_{0},\|a\| e_{0} \in \mathcal{U}$ and $\delta_{t_{a}} \circ T=\epsilon_{a} f_{a}$ on $\mathcal{U}$ we have

$$
\begin{aligned}
\|\widetilde{T}(a)\|_{L} \geq\left|\widetilde{T}(a)\left(t_{a}\right)\right| & =\left|T\left(a+\|a\| e_{0}\right)\left(t_{a}\right)-T\left(\|a\| e_{0}\right)\left(t_{a}\right)\right| \\
& =\left|\epsilon_{a}\left(f_{a}\left(a+\|a\| e_{0}\right)-f_{a}\left(\|a\| e_{0}\right)\right)\right| \\
& =\left|f_{a}(a)\right|=\|a\| .
\end{aligned}
$$

Therefore, for each smooth point $a \in \mathcal{X},\|\widetilde{T}(a)\|_{L}=\|a\|$. Hence in the case where $\mathcal{X}$ is separable, since the set of smooth points is dense in $\mathcal{X}$ and $\widetilde{T}$ is linear we conclude that $\widetilde{T}$ is an isometry from $\mathcal{X}$ into $C_{\mathbb{R}}(L)$.

Case 2: Now we discuss the general case that $\mathcal{X}$ is not necessarily separable.

The argument for this case is similar to the one given in [17]. Using the above argument, for every separable closed subspace $X$ of $\mathcal{X}$, since $T_{X}=\left.T\right|_{\mathcal{U} \cap X}$ : 
$\mathcal{U} \cap X \longrightarrow C_{\mathbb{R}}(K)$ is an isometry, there exists a closed subset $L(X) \subseteq K$, and a linear isometry $\widetilde{T}_{X}: X \rightarrow C_{\mathbb{R}}(L(X))$ such that $\widetilde{T}_{X}(u)=\left.T(u)\right|_{L(X)}$ for all $u \in \mathcal{U} \cap X$. We recall that, by the above argument, $\widetilde{T}_{X}(a)=\left.\left(T\left(a+\|a\| e_{0}\right)-T\left(\|a\| e_{0}\right)\right)\right|_{L(X)}$ holds for all $a \in X$.

If $X_{1}$ and $X_{2}$ are two separable closed subspaces of $\mathcal{X}$, then the closed subspace $\overline{X_{1}+X_{2}}$ of $\mathcal{X}$ containing $X_{1} \cup X_{2}$, is a separable closed subspace of $\mathcal{X}$ and $L\left(\overline{X_{1}+X_{2}}\right) \subseteq L\left(X_{1}\right) \cap L\left(X_{2}\right)$. Therefore, the family

$$
\{L(X): X \text { is a closed separable subspace of } \mathcal{X}\}
$$

has finite intersection property, and thus the intersection

$$
L=\cap\{L(X): X \text { is a closed separable subspace of } \mathcal{X}\} \text {, }
$$

is a nonempty closed subset of $K$. Let $Q: C_{\mathbb{R}}(K) \longrightarrow C_{\mathbb{R}}(L)$ denote the restriction map and $\widetilde{T}: \mathcal{X} \longrightarrow C_{\mathbb{R}}(L)$ be defined by $\widetilde{T}(a)=Q \circ T\left(a+\|a\| e_{0}\right)-Q \circ T\left(\|a\| e_{0}\right)$, $a \in \mathcal{X}$. Then since $\left.\widetilde{T}\right|_{X}=\widetilde{T}_{X}$ for each closed separable subspace $X$ of $\mathcal{X}$, it follows that $\widetilde{T}$ is linear and $\widetilde{T}(u)=\left.T(u)\right|_{L}$ holds for all $u \in \mathcal{U}$. So it suffices to show that $\widetilde{T}$ is an isometry.

Clearly, for each element $a \in \mathcal{X},\|\widetilde{T}(a)\|_{L} \leq\|a\|$. On the other hand, for each closed separable subspace $X$ of $\mathcal{X}$ containing $a$, there exists $t_{X} \in L(X) \subset K$ such that $\left|\widetilde{T}_{X}(a)\left(t_{X}\right)\right|=\mid \widetilde{T}_{X}(a)\left\|_{L(X)}=\right\| a \|$. Choosing a limit point $t_{0} \in K$ of the set

$$
\left\{t_{X}: X \text { is a closed separable subspace of } \mathcal{X} \text { containing } a\right\}
$$

we conclude that $\left|\widetilde{T}(a)\left(t_{0}\right)\right|=\mid \widetilde{T}(a)\left\|_{L}=\right\| a \|$. So we need only to show that $t_{0} \in L$. Assume on the contrary that $t_{0} \notin L\left(X_{0}\right)$ for some closed separable subspace $X_{0}$ of $\mathcal{X}$ containing $a$. Then there exists a neighborhood $V$ of $t_{0}$ such that $V \cap L\left(X_{0}\right)=\varnothing$. Being $t_{0}$ a limit point, there exists closed separable subspace $Y \supseteq X_{0}$ such that $t_{Y} \in V$, which is impossible since $t_{Y} \in L(Y) \subseteq L\left(X_{0}\right)$. Thus $t_{0} \in L$ and $\left|\widetilde{T}(a)\left(t_{0}\right)\right|=\|a\|$, that is $\widetilde{T}$ is an isometry.

We should note that for a complex unital Banach algebra $A$, the general groups $A^{-1}$ of invertible elements of $A$ and $\exp (A)=\left\{\Pi_{i=1}^{n} \exp \left(x_{i}\right): x_{1}, \ldots, x_{n} \in A\right.$, $n \in \mathbb{N}\}$ satisfy the hypotheses of the above theorem.

Corollary 3.3. Under the hypotheses of Theorem 3.2, if $K$ is a compact metric space, then there exists a linear isometry $\widetilde{S}: \mathcal{X} \longrightarrow C_{\mathbb{R}}(K)$ such that $\left.\widetilde{S} u\right|_{L}=\left.(T u-g)\right|_{L}$ and $(\widetilde{S} u)(K) \subseteq \operatorname{co}((T u-g)(L))$ for all $u \in \mathcal{U}$, where $L$ is a nonempty closed subset of $K$ and $g \in C_{\mathbb{R}}(K)$.

Proof. Let $g \in C_{\mathbb{R}}(K), L \subseteq K$ and linear isometry $\widetilde{T}: \mathcal{X} \longrightarrow C_{\mathbb{R}}(L)$ be as in the above theorem. Then since $K$ is metrizable, it follows from Borsuk-Dugundji Theorem ([14, Proposition 21.1.4]) that there exists a linear isometry $S: C_{\mathbb{R}}(L) \longrightarrow$ $C_{\mathbb{R}}(K)$ such that $\left.S f\right|_{L}=f$ and $S f(K) \subseteq \operatorname{co}(f(L))$ for all $f \in C_{\mathbb{R}}(L)$. Hence the isometry $\widetilde{S}=S \circ \widetilde{T}: \mathcal{X} \longrightarrow C_{\mathbb{R}}(K)$ has the desired properties. 
We now obtain an extension property for isometries into strictly convex real Banach spaces or into real Banach spaces with additional assumptions on the range of the isometry. We recall that for a real or complex normed space $\mathcal{X}, i_{\mathcal{X}}$ is the isometrical embedding from $\mathcal{X}$ to $C_{\mathbb{R}}\left(\mathcal{X}_{1}^{*}\right)$ or $C\left(\mathcal{X}_{1}^{*}\right)$. To prove next theorem we use the following two lemmas:

Lemma 3.4. Let $\mathcal{U}$ be a nonempty subset of a real normed space $\mathcal{X}, \mathcal{Y}$ be a strictly convex real normed space and $T: \mathcal{U} \rightarrow \mathcal{Y}$ be an isometry. Then for each $a, b \in \mathcal{U}$, with $\frac{a+b}{2} \in \mathcal{U}$ we have

$$
T\left(\frac{a+b}{2}\right)=\frac{T(a)+T(b)}{2} .
$$

Proof. Let $a, b \in \mathcal{U}$. Then since $T$ is an isometry

$$
\left\|T\left(\frac{a+b}{2}\right)-T(a)\right\|=\left\|\frac{a+b}{2}-a\right\|=\frac{1}{2}\|a-b\|=\frac{1}{2}\|T(a)-T(b)\|
$$

and similarly,

$$
\left\|T\left(\frac{a+b}{2}\right)-T(b)\right\|=\frac{1}{2}\|T(a)-T(b)\| .
$$

Hence by the uniqueness property in strictly convex normed spaces we have $T\left(\frac{a+b}{2}\right)=\frac{T(a)+T(b)}{2}$.

The following Mazur-Ulam type theorem has been proved in [6]:

Lemma 3.5. [6, Lemma 2.1] Let $\mathcal{X}$ and $\mathcal{Y}$ be real normed spaces and $\mathcal{U}$ and $\mathcal{V}$ be nonempty open subsets of $\mathcal{X}$ and $\mathcal{Y}$, respectively and let $T: \mathcal{U} \longrightarrow \mathcal{V}$ be a surjective isometry. If $a, b \in \mathcal{U}$ satisfy $t a+(1-t) b \in \mathcal{U}$ for every $t \in[0,1]$, then

$$
T\left(\frac{a+b}{2}\right)=\frac{T(a)+T(b)}{2} .
$$

Theorem 3.6. Let $\mathcal{X}$ be a real normed space and $\mathcal{U}$ be a subset of $\mathcal{X}$ with a nonempty interior such that $\mathbb{R} \backslash\{0\} \mathcal{U} \subseteq \mathcal{U}$. Let $\mathcal{Y}$ be a real Banach space and $T: \mathcal{U} \longrightarrow \mathcal{Y}$ be an isometric embedding. Assume that either

(i) $\mathcal{Y}$ is strictly convex, or

(ii) $\mathcal{U}$ and $T(\mathcal{U})$ are open, $\mathbb{R} \backslash\{0\} T(\mathcal{U}) \subseteq T(\mathcal{U})$ and $\mathcal{U}$ contains a nonzero element $e_{0}$ such that $i(x)=0$ on $M\left(i\left(e_{0}\right)\right)$ implies that $x=0$ for all $x \in \mathcal{X}$.

Then there exists $y_{0} \in \mathcal{Y}$ and a linear isometry $\widetilde{T}: \mathcal{X} \longrightarrow \mathcal{Y}$ (which is surjective in case (ii)) such that $\widetilde{T}(u)=T(u)-y_{0}$ for every $u \in \mathcal{U}$.

Proof. If 0 is an interior point of $\mathcal{U}$, then $\mathcal{U}=\mathcal{X}$ and the theorem is trivial. So we can assume that $\mathcal{U}$ has a nonzero interior point. Fixing a nonzero interior point $e_{0} \in \mathcal{U}$ (with additional mentioned property in case (ii)), there exists $r_{0}>0$ such that the closed ball $B_{r_{0}}\left(e_{0}\right)=\left\{x \in \mathcal{U}:\left\|x-e_{0}\right\| \leq r_{0}\right\}$ is contained in $\mathcal{U}$. As in Theorem 3.2 we can assume that $r_{0}=1,0 \in \mathcal{U}$ (such that $\mathcal{U} \backslash\{0\}$ and $T(\mathcal{U}) \backslash\{0\}$ are open in case (ii)) and $T(0)=0$. As before, we define $\widetilde{T}: \mathcal{X} \longrightarrow \mathcal{Y}$ by $\widetilde{T}(a)=T\left(a+\|a\| e_{0}\right)-T\left(\|a\| e_{0}\right)$ for all $a \in \mathcal{X}$. We should note that for each 
nonzero $a \in \mathcal{X}$ we have $\frac{a}{\|a\|}+e_{0} \in B_{1}\left(e_{0}\right) \subseteq \mathcal{U}$ and consequently $a+\|a\| e_{0} \in \mathcal{U}$ for all $a \in \mathcal{X}$.

Since $0 \in \mathcal{U}$ and for each $u \in \mathcal{U}$ we have $-u \in \mathcal{U}$ it follows from Lemma 3.4 that in case (i), $0=T(0)=T(u)+T(-u)$, that is $T(-u)=-T(u)$. In case (ii), using Lemma 3.5 for nonzero elements $u$ and $-u+\frac{1}{n} u, n \in \mathbb{N}$, in $\mathcal{U}$ we have the same equality. On the other hand, for each $u \in \mathcal{U}$, since $\frac{u}{n} \in \mathcal{U}$ for all $n \in \mathbb{N}$, using Lemmas 3.4 and 3.5 once again, we get $T\left(\frac{u-\frac{u}{n}}{2}\right)=\frac{T(u)-T\left(\frac{u}{n}\right)}{2}$ for all $n \in \mathbb{N}$. Hence tending $n \rightarrow \infty$ since $T$ is continuous we get $T(u)=2 T\left(\frac{u}{2}\right)$. Continuity of $T$ easily implies that for each $r \in \mathbb{R}$ and $u \in \mathcal{U}, T(r u)=r T(u)$.

We should note that for each $u, v \in \mathcal{U}$ with $\frac{u+v}{2} \in \mathcal{U}$ (equivalently $u+v \in \mathcal{U}$ ) we have, by Lemma 3.4, in case (i) that $T\left(\frac{u+v}{2}\right)=\frac{T(u)+T(v)}{2}$ and consequently in this case, for such $u, v \in \mathcal{U}$ we have

$$
T(u+v)=T(u)+T(v) .
$$

Using Lemma 3.5 in case (ii) we have $T\left(\frac{u+v}{2}\right)=\frac{T(u)+T(v)}{2}$ for all $u, v \in \mathcal{U}$ with $t u+(1-t) v \in \mathcal{U} \backslash\{0\}, t \in[0,1]$. In particular, since for each nonzero $a \in \mathcal{U}$ in case (i) and $a \in \mathbb{R}^{+} B_{1}\left(e_{0}\right)$ in case (ii), $a+\|a\| e_{0},\|a\| e_{0} \in \mathbb{R}^{+} B_{1}\left(e_{0}\right) \subseteq \mathcal{U}$ we have $T\left(a+\|a\| e_{0}\right)=T(a)+T\left(\|a\| e_{0}\right)$ and consequently in case (i)

$$
\widetilde{T}(a)=T\left(a+\|a\| e_{0}\right)-T\left(\|a\| e_{0}\right)=T(a) \quad(a \in \mathcal{U})
$$

and in case (ii)

$$
\widetilde{T}(a)=T\left(a+\|a\| e_{0}\right)-T\left(\|a\| e_{0}\right)=T(a) \quad\left(a \in \mathbb{R}^{+} B_{1}\left(e_{0}\right)\right) .
$$

Hence in case (i), $\widetilde{T}$ is an extension of $T$.

Now in both cases since for each nonzero $a \in \mathcal{X}$ and $r \geq\|a\|, a+r e_{0}$, $\|a\| e_{0} \in \mathbb{R}^{+} B_{1}\left(e_{0}\right)$, using the same argument as in Theorem 3.2 we can show that $\widetilde{T}$ is linear. As before, in both cases, $\widetilde{T}$ is an isometry. Indeed, for each $a \in \mathcal{X}$, we have

$$
\|\widetilde{T}(a)\|=\left\|T\left(a+\|a\| e_{0}\right)-T\left(\|a\| e_{0}\right)\right\|=\|a+\| a\left\|e_{0}-\right\| a\left\|e_{0}\right\|=\|a\| .
$$

which shows, by the real-linearity of $\widetilde{T}$ that $\widetilde{T}$ is an isometry.

To complete the proof, it suffices to show that in case (ii), $\widetilde{T}$ is surjective and $\widetilde{T}(u)=T(u)$ for all $u \in \mathcal{U}$. Set $\mathcal{V}=T(\mathcal{U} \backslash\{0\})$. We note that since in case (ii), $\mathcal{V}$ is assumed to be open, there exists $s_{0} \leq 1$ such that $B_{s_{0}}\left(T\left(e_{0}\right)\right) \subseteq \mathcal{V}$. Hence $T\left(B_{1}\left(e_{0}\right)\right) \supseteq B_{s_{0}}\left(T\left(e_{0}\right)\right)$. Therefore, for a point $y \in \mathcal{V}$, since $\frac{s_{0} y}{\|y\|}+T\left(e_{0}\right) \in$ $B_{s_{0}}\left(T\left(e_{0}\right)\right)$ there exists a point $x \in B_{1}\left(e_{0}\right)$ such that $\frac{s_{0} y}{\|y\|}+T\left(e_{0}\right)=T(x)$. Since, by (3.9), $T\left(e_{0}\right)=\widetilde{T}\left(e_{0}\right)$ and $T(x)=\widetilde{T}(x)$, the linearity of $\widetilde{T}$ implies that $y \in \widetilde{T}(\mathcal{X})$, that is $\widetilde{T}$ is surjective.

Given $u \in \mathcal{U} \backslash\{0\}$, set $b=T(u)$. Then since $\widetilde{T}$ is surjective, there exists $v \in \mathcal{X}$ with $\widetilde{T}(v)=b$. Since $u+\|u\| e_{0} \in \mathbb{R}^{+} B_{1}\left(e_{0}\right)$ we have $\widetilde{T}\left(u+\|u\| e_{0}\right)=$ $T\left(u+\|u\| e_{0}\right)$, by (3.9), and hence

$$
\begin{aligned}
\|u\|\left\|e_{0}\right\| & =\left\|T\left(u+\|u\| e_{0}\right)-T(u)\right\|=\left\|\widetilde{T}\left(u+\|u\| e_{0}\right)-\widetilde{T}(v)\right\| \\
& =\|u+\| u\left\|e_{0}-v\right\|=\left\|i_{\mathcal{X}}\left(u+\|u\| e_{0}-v\right)\right\| \mathcal{X}_{1}^{*}
\end{aligned}
$$


Hence for each $l \in \mathcal{X}_{1}^{*}, i_{\mathcal{X}}(u-v)(l)$ is in the closed disk $B_{\|u\|\left\|e_{0}\right\|}\left(\|u\| i_{\mathcal{X}}\left(e_{0}\right)(l)\right)$. Using the same argument for $-u+\|u\| e_{0}$, we conclude that for each $l \in \mathcal{X}_{1}^{*}$, $i_{\mathcal{X}}(u-v)(l)$ is in the closed disk $B_{\|u\|\left\|e_{0}\right\|}\left(-\|u\| i_{\mathcal{X}}\left(e_{0}\right)(l)\right)$. Therefore, for each $l \in M\left(i_{\mathcal{X}}\left(e_{0}\right)\right), i_{\mathcal{X}}(u-v)(l)=0$, that is $i_{\mathcal{X}}(u-v)=0$ on $M\left(i_{\mathcal{X}}\left(e_{0}\right)\right)$ and consequently $u=v$, by the assumption on $M\left(i_{\mathcal{X}}\left(e_{0}\right)\right)$. Therefore, $\widetilde{T}(u)=T(u)$, as desired.

Remark. a) We should note that the assumptions $\mathbb{R} \backslash\{0\} T(\mathcal{U}) \subset T(\mathcal{U})$ and existence of a point $e_{0} \in \mathcal{U}$ with the mentioned property in case (ii) of the above theorem, have been used to prove the surjectivity of $\widetilde{T}$ and equality $\widetilde{T}(u)=T(u)$ for all $u \in \mathcal{U}$. However, without these assumptions $\widetilde{T}$ is a linear isometry with $\widetilde{T}(a)=T(a)$ for all $a \in \mathbb{R}^{+} B_{1}\left(e_{0}\right)$.

b) The proof of case (ii) in the above theorem can also be applied for complex normed spaces with the same hypothesis on $M\left(i_{\mathcal{X}}\left(e_{0}\right)\right)$. In this case $\widetilde{T}$ is a reallinear isometry. As we mentioned before, in subspaces of the form $A x_{0}$ where $A$ is a closed commutative semisimple unital subalgebra of a unital complex Banach algebra $B$ and $x_{0} \in B$ is invertible with $\left\|x_{0}\right\|=\left\|x_{0}^{-1}\right\|=1$, the maximum set of $i_{A x_{0}}\left(x_{0}\right)$ has the desired property. Hence case (ii) of the above theorem, can be applied for appropriate subsets $\mathcal{U}$ of $A x_{0}$.

Next corollary is immediate from Theorem 3.2 and case(i) of the above theorem.

Corollary 3.7. Let $B$ be a unital Banach algebra, $A$ be a closed unital subalgebra of $B$ and $x_{0} \in B$ be invertible. If $G$ is an open subgroup of invertible elements of $A$ and $T: G x_{0} \longrightarrow \mathcal{Y}$ is an isometric embedding, where $\mathcal{Y}$ is either $C_{\mathbb{R}}(K)$ for some compact Hausdorff space $K$ or is a strictly convex real Banach space, then there exists $y_{0} \in \mathcal{Y}$ and a real-linear isometry $\widetilde{T}: A x_{0} \longrightarrow \mathcal{Y}$ such that $\widetilde{T}\left(a x_{0}\right)=T\left(a x_{0}\right)-y_{0}$, for all $a \in G$.

We should note that an interesting generalization of Mazur-Ulam theorem for isometric embeddings $T: \mathcal{X} \longrightarrow \mathcal{Y}$ between real normed spaces $\mathcal{X}$ and $\mathcal{Y}$ was given in [3] and then imposing an additional assumption on the range (weaker than surjectivity assumption) a Mazur-Ulam type theorem was given in [4]. In the next theorem and corollary we give similar results for the case where the domain of $T$ is an appropriate subset of $\mathcal{X}$.

Theorem 3.8. Let $\mathcal{X}$ be a real normed space, $\mathcal{U}$ be a nonempty open subset of $\mathcal{X}$ such that $\mathbb{R} \backslash\{0\} \mathcal{U} \subseteq \mathcal{U}$ and $\mathcal{Y}$ be a real Banach space. Let $T: \mathcal{U} \rightarrow \mathcal{Y}$ be an isometric embedding. Then there exists a surjective linear map $S: \mathcal{Y} \longrightarrow \operatorname{span} \mathcal{U}$ such that $S(T(u))=u$ for all $u \in \mathcal{U}$ and the restriction of $S$ to span $T(\mathcal{U})$ has norm one.

Proof. The proof of this theorem is a minor modification of [3, Main theorem]. As before we can assume that $0 \in \mathcal{U}, \mathcal{U} \backslash\{0\}$ is open and $T(0)=0$. Furthermore, we can extend $T$ to an isometry, denoted again by $T$ from the closure $\mathcal{V}$ of $\mathcal{U}$ to $\mathcal{Y}$.

Let $\mathcal{X}^{\prime}, \mathcal{Y}^{\prime}$ stand for the linear span of $\mathcal{U}$ and $T(\mathcal{U})$, respectively. If $\operatorname{dim} \mathcal{X}^{\prime}=1$, then since $\mathbb{R} \mathcal{V} \subseteq \mathcal{V}$ it follows that $\mathcal{V}=\mathcal{X}^{\prime}$ and so by [3, Lemma 1] there exists a linear map $S: \mathcal{Y} \rightarrow \mathcal{X}^{\prime}$ such that $\|S\|=1, S(T(x))=x$ for all $x \in \mathcal{X}^{\prime}$.

Now assume that $\mathcal{X}^{\prime}$ is finite dimensional. Then since $\mathcal{X}^{\prime}$ is a separable Banach space, the set of all smooth points of $\mathcal{X}^{\prime}$ is a dense subset of $\mathcal{X}^{\prime}$ and as before the 
set of all smooth points of $\mathcal{X}^{\prime}$ contained in $\mathcal{V}$ is dense in $\mathcal{V}$. As, in this case, $\mathcal{X}^{\prime}=\operatorname{span} \mathcal{U}=\operatorname{span} \mathcal{V}$ it follows that there are smooth points $a_{1}, \ldots, a_{n}$ in $\mathcal{V}$ such that $\mathcal{X}^{\prime}=\operatorname{span}\left\{a_{1}, \ldots, a_{n}\right\}$. This easily implies that $\left\{f_{a_{1}}, \ldots, f_{a_{n}}\right\}$ is a basis for $\left(\mathcal{X}^{\prime}\right)^{*}$. For every $1 \leq i \leq n$, using the previous case, we can choose $s_{i} \in \mathcal{Y}^{*}$ with $\left\|s_{i}\right\|=1$ such that $s_{i}\left(T\left(r a_{i}\right)\right)=r\left\|a_{i}\right\|$ for all $r \in \mathbb{R}$. Hence by Lemma 3.1, for each $1 \leq i \leq n$ and $v \in \mathcal{V}$ we have $s_{i}(T(v))=f_{a_{i}}(v)$. Now consider the map $S: \mathcal{Y} \longrightarrow \mathcal{X}^{\prime}$ defined by

$$
S(y)=G^{-1}\left(s_{1}(y), \ldots, s_{n}(y)\right) \quad(y \in \mathcal{Y}),
$$

where $G: \mathcal{X}^{\prime} \rightarrow \mathbb{R}^{n}$ is the linear isomorphism defined by $G(x)=\left(f_{a_{1}}(x), \ldots\right.$, $\left.f_{a_{n}}(x)\right)$. Then $S$ is a linear map whose restriction to $\mathcal{Y}^{\prime}$ is continuous and for each $v \in \mathcal{V}$,

$$
S(T(v))=G^{-1}\left(s_{1}(T(v)), \ldots, s_{n}(T(v))\right)=G^{-1}\left(f_{a_{1}}(v), \ldots, f_{a_{n}}(v)\right)=v
$$

By a similar argument as in [3, Lemma 4], we get $\left\|\left.S\right|_{\mathcal{Y}^{\prime}}\right\|=1$ and then the same argument as in [3, Main Theorem] can be applied to complete the proof for the general case that $\mathcal{X}^{\prime}$ is not necessarily of a finite dimension.

Corollary 3.9. Under the hypotheses of the above theorem, if for every unit vector $y \in \operatorname{span} T(\mathcal{U})$ there exist $a, b \in \mathcal{U}$ and $\lambda \in \mathbb{R}$ such that

$$
\|y-\lambda(T(a)-T(b))\|<\frac{1}{2}
$$

then there exists $y_{0} \in \mathcal{Y}$ and a (real) linear isometry $\widetilde{T}: \mathcal{X} \longrightarrow \mathcal{Y}$ such that $\widetilde{T}(u)=$ $T(u)-y_{0}$ for every $u \in \mathcal{U}$.

Proof. Assuming $0 \in \mathcal{U}, \mathcal{U} \backslash\{0\}$ is open and $T(0)=0$ and extending $T$ to an isometry, denoted again by $T$ from the closure $\mathcal{V}$ of $\mathcal{U}$ to $\mathcal{Y}$, we define $\widetilde{T}: \mathcal{X} \rightarrow \mathcal{Y}$ by $\widetilde{T}(0)=0$ and $\widetilde{T}(a)=T\left(a+\|a\| e_{0}\right)-T\left(\|a\| e_{0}\right)$ for $a \neq 0$ which is clearly well-defined.

By the above theorem there exists a surjective linear map $S: \mathcal{Y} \longrightarrow \operatorname{span} \mathcal{U}$ whose restriction to $\operatorname{span} T(\mathcal{U})$ is of norm one and $S(T(u))=u$ for all $u \in \mathcal{U}$. The hypothesis on the unit vectors in span $T(\mathcal{U})$ easily implies that the restriction of $S$ to the linear span $\mathcal{Y}^{\prime}$ of $T(\mathcal{U})$ is injective (see the proof of unique theorem in [4]).

Let $\mathcal{X}^{\prime}$ be the linear span of $\mathcal{U}$. As $S^{-1}: \mathcal{X}^{\prime} \rightarrow \mathcal{Y}^{\prime}$ is a (real) linear map and $S(T(u))=u$ for all $u \in \mathcal{U}$ which implies the same equality for all $u \in \mathcal{V}$, we conclude that $T(r u)=r T(u)$ holds for all $r \in \mathbb{R}$ and $u \in \mathcal{V}$. Moreover, if $a, b \in \mathcal{V}$ such that $a+b \in \mathcal{V}$, then $T(a+b)=T(a)+T(b)$. In particular, for each $a \in \mathcal{U}$, since $a+\|a\| e_{0},-\|a\| e_{0} \in \mathcal{U} \subset \mathcal{V}$ we have $\widetilde{T}(a)=T\left(a+\|a\| e_{0}\right)-T\left(\|a\| e_{0}\right)=$ $T(a)$.

Now the same argument as in the proof of Theorem 3.2, shows that $\widetilde{T}$ is a (real) linear isometry.

Acknowledgement. The authors would like to thank the referee for his/her helpful comments. 


\section{References}

[1] J. A. Baker, Isomtries in normed spaces, Amer. Math. Monthly, 78 (1971), 655658.

[2] Z. Chrzynski, Sur les transformations isometriques des espaces du type F, Studia Math. 13 (1953), 94-121.

[3] T. Figiel, On nonlinear isometric embeddings of normed linear spaces, Bull. Acad. Polon. Sci. Math. Astro. Phys. 16 (1968), 185-188.

[4] T. Figiel, P. Šemrl, J. Väisälä, Isometries of normed spaces, Colloq. Math. 92, (2002), 153-154.

[5] O. Hatori, Algebraic properties of isometries between groups of invertible elements in Banach algebras, J. Math. Anal. Appl. 376 (2011), 84-93.

[6] O. Hatori, Isometries between groups of invertible elements in Banach algebras, Studia Math. 194 (2009), 293-304.

[7] O. Hatori, K. Kobayashi, T. Miura and S-E. Takahasi, Reflections and generalizations of the Mazur-Ulam theorem, Rocky Mountain J. Math., 42 (2012), 117150.

[8] R. Liu, A note on extension of isometric embedding from a Banach space E into the universal space $l_{\infty}(\Gamma)$, J. Math. Anal. Appl. 363 (2010), 220-224.

[9] P. Mankiewiz, On extension of isometries in normed spaces, Bull. Acad. Polon. Sci. 20 (1972), 367-371.

[10] S. Mazur, S. Ulam, Sur les transformations isometriques d'espaces vectoriels normes, C. R. Acad. Sci. Paris 194 (1932), 946-948.

[11] A. R. Pears, Dimension Theory of General Spaces, Cambridge, CUP, 1975

[12] S. Rolewicz, A Generalization of the Mazur-Ulam theorem, Studia Math. 31 (1968), 501-505.

[13] S. Rolewicz, Metric Linear Spaces, Reidel and PWN, Dordrecht and Warszawa, 1985.

[14] Z. Semadeni, Banach Spaces of Continuous Functions, PWN, Warszawa, 1971.

[15] D. Tingley, Isometries of the unit sphere, Geom. Dedicata 22 (1987), 371-378.

[16] J. Väisälä, A proof of the Mazur-Ulam theorem, Amer. Math. Monthly 110 (2003) 633-635.

[17] R. Villa, Isometric embedding into spaces of continuous functions, Studia Math. 129 (1998), 197-205.

[18] A. Vogt, Maps which preserve equality of distance, Studia Math. 45 (1973) 43-48. 
[19] J. Wang, On extension of isometries between unit spheres of $A L_{p}$-spaces $(1<p<$ $\infty)$, Proc. Amer. Math. Soc. 132 (2004), 2899-2909.

[20] X. Yang, On extension of isometries between unit spheres of $L_{p}(\mu)$ and $L_{p}(\nu, H)$ $(1<p \neq 2, H$ is a Hilbert space), J. Math. Anal. Appl. 323 (2006), 985-992.

Department of Pure Mathematics,

Faculty of Mathematical Sciences,

Tarbiat Modares University,

Tehran, 14115-134, Iran

Email addresses: h.khodaiemehr@modares.ac.ir, sady@modares.ac.ir 